Original Paper http://ajol.info/index.php/ijbcs $\quad$ http://indexmedicus.afro.who.int

\title{
Etude ethnobotanique des plantes alimentaires utilisées en période de soudure dans les régions Sud du Mali
}

\author{
Nouhoum DIARRA ${ }^{1}$, Adiaratou TOGOLA ${ }^{2 *}$, Adama DENOU ${ }^{3}$, Merlin WILLCOX $^{4}$, \\ Cheickna DAOU ${ }^{5}$ et Drissa DIALLO ${ }^{2,3}$ \\ ${ }^{1}$ Faculté des Sciences et des Techniques, Université des Sciences, des Techniques et des Technologies de \\ Bamako; BPE 3206 Badalabougou Bamako, Mali. \\ ${ }^{2}$ Département de Médecine Traditionnelle, Institut National de Recherche en Santé Publique, \\ BP 1746 Hippodrome Bamako, Mali. \\ ${ }^{3}$ Faculté de Pharmacie, Université des Sciences, des Techniques et des Technologies de Bamako, \\ BP 1805 Point G, Bamako, Mali. \\ ${ }^{4}$ Nuffield Department of Primary Care Health Sciences, University of Oxford, Radcliffe Observatory Quarter, \\ Woodstock Road, Oxford OX 2 6GG, United Kingdom. \\ ${ }^{5}$ Institut des Sciences Appliquées, Université des Sciences, des Techniques et des Technologies de Bamako \\ (USTTB) Colline de Badalabougou, BP E 3206, Bamako, Mali. \\ *Auteur correspondant ; E-mail: togola_adia@hotmail.com; adiatog@yahoo.fr \\ : Tel : (+223) 75344429 (+223) 66790276,
}

\section{REMERCIEMENTS}

Ce travail a été financièrement supporté par le Programme de Formation des Formateurs de l'Université de Bamako, Mali.

\section{RÉSUME}

La période de soudure est celle pendant laquelle les stocks de céréales sont épuisés et les prochaines récoltes ne sont pas encore prêtes. Au Mali (Afrique de l'Ouest), elle varie d'août à septembre. Pendant cette période, les communautés locales utilisent les plantes sauvages comme aliment. L'objectif de la présente étude était de recenser les plantes sauvages alimentaires consommées dans les régions Sud du Mali (Kayes, Koulikoro, Sikasso et Ségou) pendant cette période. Des enquêtes ethnobotaniques utilisant les méthodes du focus group, d'enquête individuelle et d'interviews semi-structurées ont été menées. Au total, 454 personnes dont 338 hommes et 116 femmes soit respectivement 74,4\% et 25,6\% ont été interrogées. Les jeunes (15-30 ans), cultivateurs et Bambara étaient les plus nombreux. 87 plantes alimentaires appartenant à 44 familles ont été identifiées; les fruits de 56 plantes $(62,2 \%)$, les feuilles de 43 plantes $(47,8 \%)$ et les graines de 10 plantes (11,5\%) sont consommées comme aliments pendant les périodes de soudure. Parkia biglobosa, Adansonia digitata et Vitellaria paradoxa sont les plantes les plus utilisées soit seules ou dans des plats comme additifs. Certaines de ces plantes servent également comme médicament et comme source de revenus pour les communautés locales.

(C) 2016 International Formulae Group. All rights reserved.

Mots clés: Plantes sauvages alimentaires, enquête ethnobotanique, période de soudure, Sud-Mali. 


\title{
Ethnobotanical study of food plants used in the period of soldering in the southern regions of Mali
}

\begin{abstract}
The lean period is the one during which grain stocks are depleted and the next harvest is not yet ready. In Mali (West Africa), it varies from August to September. During this period, local communities use wild plants as food. The objective of this study was to identify wild food plants consumed in the southern regions of Mali (Kayes, Koulikoro, Segou and Sikasso) during this period. Ethnobotanical surveys using focus group methods, individual survey and semi-structured interviews were conducted. A Total of 454 respondents including $338(74.4 \%)$ men and $116(25.6 \%)$ women were interviewed. Yong farmers (15-30 years) from Bambara ethic group were the most numerous. 87 food plants belonging to 44 families have been identified; the fruit of $56(62.2 \%)$ plants, the leaves of $43(47.8 \%)$, and the seeds of 10 plants $(11.5 \%)$ were consumed as food during lean periods. Parkia biglobosa, Adansonia digitata and Vitellaria paradoxa are the most used plants either alone or in dishes such as additives. Some of these plants are also used as a medicine and as a source of income for local communities.
\end{abstract}

(C) 2016 International Formulae Group. All rights reserved.

Keywords: wild food plants, ethnobotany survey, lean period, South Mali.

\section{INTRODUCTION}

Selon la FAO, le nombre de personnes sous-alimentées, c'est-à-dire souffrant de la faim presque tous les jours, dans le monde en ce début de $\mathrm{XXI}^{\mathrm{ème}}$ siècle, est d'environ 855 millions, dont 820 millions dans les pays en développement, 25 millions dans les pays en transition et 10 millions dans les pays développés. Ainsi, dans les pays en développement, près d'une personne sur cinq est sous-alimentée; ce chiffre augmente à une personne sur trois en Afrique, (Roudart, 2007).

Des travaux de recherche ont montré l'importance des espèces végétales sauvages dans l'alimentation des hommes surtout pendant les périodes de disette et cela partout dans le monde et principalement en Afrique. En République Démocratique du Congo, une étude a montré que pendant la période de soudure, les populations vont creuser en brousse les tubercules pour les manger (Tollens, 2003). Une autre étude de la consommation de fruits de la forêt au Zimbabwe a montré que la cueillette et la consommation de fruits sauvages atteignent leur maximum lorsque les aliments tirés de plantes cultivées viennent à manquer (Lapante, 2009). Dans la vallée de la Luangwa (Zambie) les végétaux sauvages jouent un rôle important dans l'alimentation tandis qu'au
Botswana, les Bochimans San tirent de l'eau potable de nombreuses plantes, en particulier les tubercules (van Wyk et Gericke, 2007; Lapante 2009). Au Burkina Faso, au Sénégal et à travers toute l'Afrique de l'Ouest, les plantes sauvages constituent le seul recours des populations pendant les périodes de soudure et de famine (Easton et Ronald, 2000; Chastanet, 2010).

Bien que les régions sud du Mali (Kayes, Koulikoro, Sikasso et Ségou) regroupent la majeure partie de la diversité forestière du pays, aucune étude approfondie n'a été menée sur les plantes alimentaires sauvages utilisées en période de soudure.

L'objectif du présent travail était d'inventorier les plantes alimentaires utilisées en période de soudure par les populations rurales de ces régions.

\section{MATÉRIEL ET MÉTHODES \\ Choix des sites}

Dans chaque région, trois localités ont été choisies en raison des critères bioclimatiques à savoir leur situation dans la zone pré guinéenne, soudanienne et sahélienne; pour couvrir l'ensemble des formations végétales des régions du sud (Figure 1). Dans la région de Kayes, les cercles de Kita, Bafoulabé et Diéma, respectivement situés dans la zone pré 
guinéenne soudanienne et sahélienne ont été choisis. Dans la région de Koulikoro, Dioila situé dans la zone soudanienne, Kolokani et Banamba dans la zone sahélienne ont été choisis. Dans la région de Sikasso, les zones choisies sont Yanfolila et Bougouni qui se trouvent dans les zones pré- guinéenne et soudanienne et Koutiala dans les zones soudanienne et sahélienne. Dans la région de Ségou, les cercles de San et Bla sont dans la zone sahélienne et la localité de Konobougou dans le cercle de Baraouli est dans la zone soudanienne (INSTAT, 2009).

\section{Description des régions d'étude}

Les régions Sud du Mali s'étendent de Sikasso à Kayes en passant par Koulikoro et Ségou (Figure 1) avec une superficie de $343.610 \mathrm{~km}^{2}$ soit environ le tiers de la superficie nationale du Mali pour une population estimée à 9.377.291 habitants soit 2/3 de la population du pays (INSTAT, 2009). Le climat de ces régions est varié de type tropical guinéen, soudanien et sahélien, avec des écarts de températures importants. On y distingue trois saisons dont la durée varie avec la latitude; une saison sèche froide (novembre à février), une saison sèche chaude (mars à juin), et une saison pluvieuse (juillet à octobre). Les vents dominants sont la mousson qui vient du Sud et occasionne la pluie, et l'alizé chaud et sec qui vient du Nord (DRPS, 2000).

A Kayes, on note la présence d'espèces hygrophiles (Mimosa pigra, Mitragyna inermis, Anogeissus leiocarpus, Nauclea latifolia, etc.). La végétation de Koulikoro est dominée par Terminalia spp, Pseudocedrela spp, Gardenia spp et Vitellaria paradoxa. Les espèces ligneuses dominantes sont Acacia seyal, Mitragyna inermis, Anogeissus leiocarpus, Piliostigma reticulatum, Terminalia spp, Acacia adansonï, Diospiros mespiliformis. La végétation de Sikasso se caractérise par la savane parc à Vitellaria paradoxa, Daniellia oliveri, Annona senegalensis, Sclerocarya birrea. Le tapis herbacé comporte Eragrostis tremula, Loudetia togoensis. Le potentiel fourrager de la zone est élevé. Dans la région de Ségou, la végétation des zones inondées est presque totalement graminéenne avec des espèces caractéristiques comme Vetiveria nigritana, Panicum anabaptistum, Oryza sativa, Oryza longistaminata, Eragrostis barthï, Corchorus spp. En zone susceptible d'être exondée, certaines espèces ligneuses apparaissent : Terminalia spp, Pseudocedrela spp, Gardenia spp et Vitellaria paradoxa. Les espèces ligneuses dominantes sont Acacia seyal, Mitragyna inermis, Anogeissus leiocarpus, Piliostigma reticulatum, Terminalia spp, Acacia adansoniï, Diospiros mespiliformis. Le tapis herbacé est dominé par: Schoenefeldia gracilis, Panicum spp, Hyparhenia rufa et Andropogon gayanus.

\section{Administration du questionnaire \\ Dans chaque chef-lieu de cercle, les} fiches d'enquête ont été administrées soit individuellement, soit à des groupes de personnes. Les fiches d'enquêtes ont été remplies par les enquêteurs à différents endroits de la ville (marché, famille, champs, rue, etc.). Trois modes de collecte des données ont été utilisés: « Focus group » enquête groupée au cours de laquelle les fiches sont remplies durant des causeries débats; enquête individuelle au cours de laquelle l'enquêteur pose les questions à une personne et les interviews semi-structurées par groupe ont été faites suivant la méthode décrite par Wentholt et al. (2001) qui consiste à interroger les femmes et les hommes séparément. Elles ont été faites en langue locale au moyen de questions ouvertes.

Les questions portaient sur l'énumération des plantes sauvages alimentaires consommées dans les différentes zones, les parties de ces plantes (feuilles, racines, tubercules, fruits) consommées, les modes de préparation (cru ou frais, cuit, séché) et les périodes de consommation (toute l'année ou pendant la période de soudure), la disponibilité et les moyens d'approvisionnement, les moyens de récolte et de transport, la commercialisation, la disparition de certaines espèces du terroir, les rôles socio-économiques et thérapeutiques de ces plantes. L'enquête sur le terrain a duré de juin 2009 à mai 2010. Cette période a permis de situer les moments de floraison et de 
fructification de la majeure partie des plantes sauvages.

\section{RÉSULTATS}

\section{La population de l'enquête}

L'enquête a porté sur 454 personnes dont $74,4 \%$ d'hommes et $25,6 \%$ de femmes. Les résultats de la répartition de la population d'enquête par région et par sexe sont consignés dans le Tableau 1. Le pourcentage des populations échantillonnées était de $21,8 \%$ à Kayes, $27,1 \%$ à Koulikoro, 24,9\% à Sikasso et $26,2 \%$ à Ségou. L'enquête a concerné les personnes âgées de 15 à 60 ans. Globalement, la tranche d'âge de 15-30 ans était la plus représentative suivi de la tranche d'âge 46-60 dans l'ensemble des régions enquêtées. Les populations enquêtées étaient majoritairement composées de Bambara dans chacune des régions excepté Sikasso où les Senoufo étaient majoritaires (Figure 2). L'enquête a concerné plus d'ethnies à Kayes et Ségou que dans les autres régions. Les Bambaras, les Peulhs, les Malinkés ont été rencontrés dans toutes les localités échantillonnées. Les Kassonkés ont été retrouvés uniquement dans la région de Kayes, tandis que les Bozo n'ont été enquêtés que dans la région de Ségou. La population d'enquête était composée par les groupes socio-economiques suivants : les cultivateurs, les tradipraticiens, les ménagères, les éleveurs, les étudiants, les sans-emplois, les administrateurs, les commerçants, les pêcheurs et les enseignants. Les cultivateurs et les tradipraticiens de santé étaient les plus représentés dans l'échantillon enquêté. Les tradipraticiens de santé étaient majoritaires dans la région de Sikasso tandis que les cultivateurs constituaient la couche socioprofessionnelle la plus représentée dans les autres régions.

\section{Les plantes sauvages alimentaires}

Toute plante poussant d'elle-même dans la nature et dont on peut utiliser un organe dans l'alimentation est appelée plante sauvage alimentaire dans le contexte de ce travail.

$\mathrm{Au}$ total, 87 plantes sauvages alimentaires réparties entre 47 familles et 78 genres ont été recensées auprès des personnes enquêtées (Tableau 2). Les plantes les plus citées étaient Adansonia digitata, Parkia biglobosa, Combretum micranthum, Saba senegalensis et Ficus thonningii. Elles étaient largement utilisées par les populations enquêtées pour des besoins alimentaire et thérapeutique. Les fruits charnus ou secs sont les plus consommés, suivis des feuilles fraîches parfois séchées.

Tous les fruits sont consommés à l'état naturel. En général, la période de grande consommation correspond à la période de disponibilité, cependant certains fruits restent disponible ou sont récoltés pour être stockés par les populations pour préparer les périodes de pénuries alimentaires. Certains fruits sont consommés grâce à leur goût sucré et d'autres uniquement lorsqu'ils sont disponibles comme les fruits de Annona senegalensis, Vitellaria paradoxa ou Saba senegalensis. Par contre, les fruits de Cordylla pinnata immatures sont récoltés et séchés puis consommés pendant les périodes de soudure ou de disette. Le mode de préparation et de consommation varient selon les populations.

Les feuilles fraîches ou séchées sont en général consommées dans les sauces, c'est le cas de Adansonia digitata, Corchorus tridens, Cleome gynandra, Hibiscus sabdarifa, Portulaca grandiflora et les bourgeons de Cordia myxa. Les feuilles de Combretum micranthum sont utilisées comme thé. Les parties de ces plantes consommées sont représentées dans le Tableau 2. La plupart des plantes citées sont communes aux 4 régions enquêtées, certaines plantes ont même été rencontrées dans toutes les localités où l'enquête a eu lieu, c'est le cas de Adansonia digitata, Zizyphus mauritiana, Vitellaria paradoxa, Nymphaea lotus, Parkia biglobosa, Saba senegalensis, Lannea acida, Sclerocarya birrea, Balanites aegyptiaca, Tamarindus indica et Cordyla pinnata.

Les parties de plantes les plus consommées étaient les fruits $(51,3 \%)$, suivies des feuilles $(27,6 \%)$, des fleurs $(11,8 \%)$, les tubercules ou racines $(5,2 \%)$ et les graines $(3,9 \%)$. Cette chronologie a été observée dans toutes les localités enquêtées. 


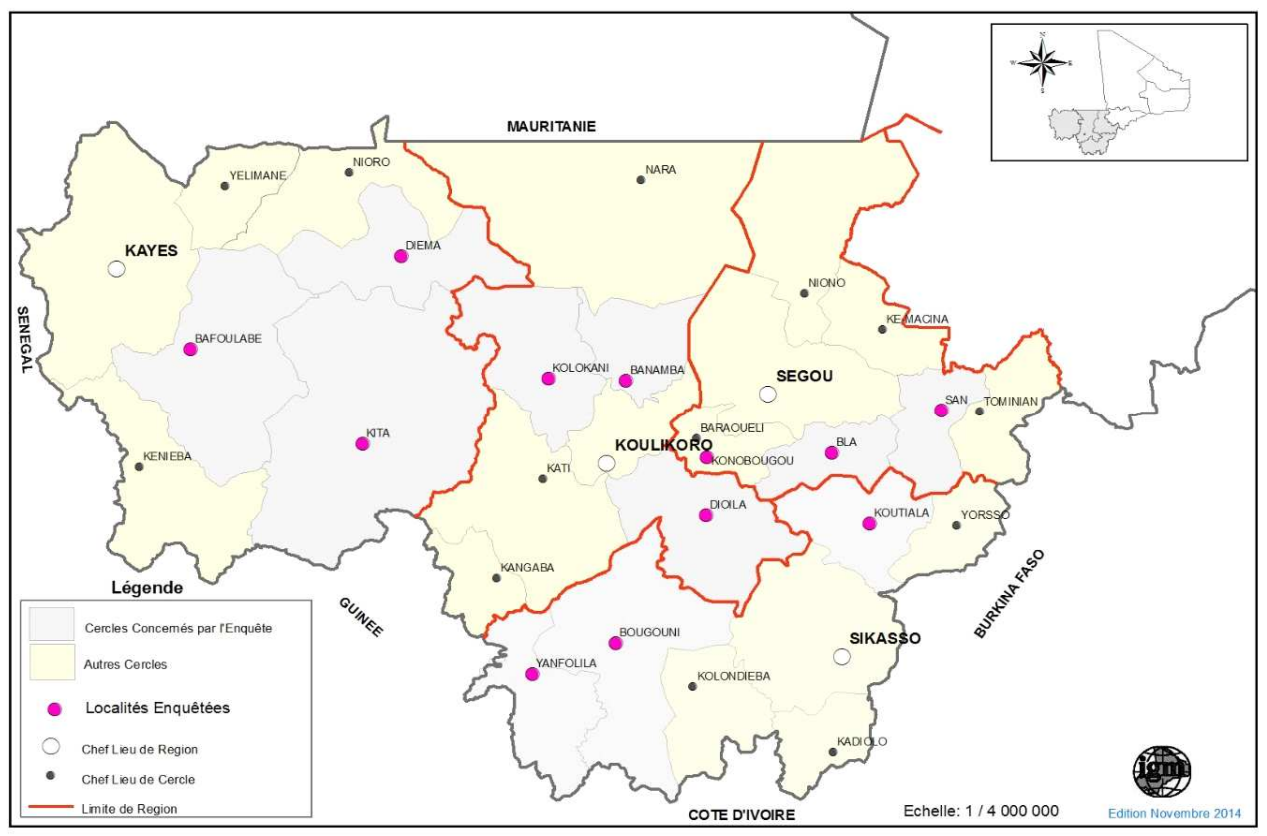

Figure 1: Carte géographique des régions Sud du Mali (Kayes, Koulikoro, Ségou et Sikasso) montrant les zones d'enquêtes.
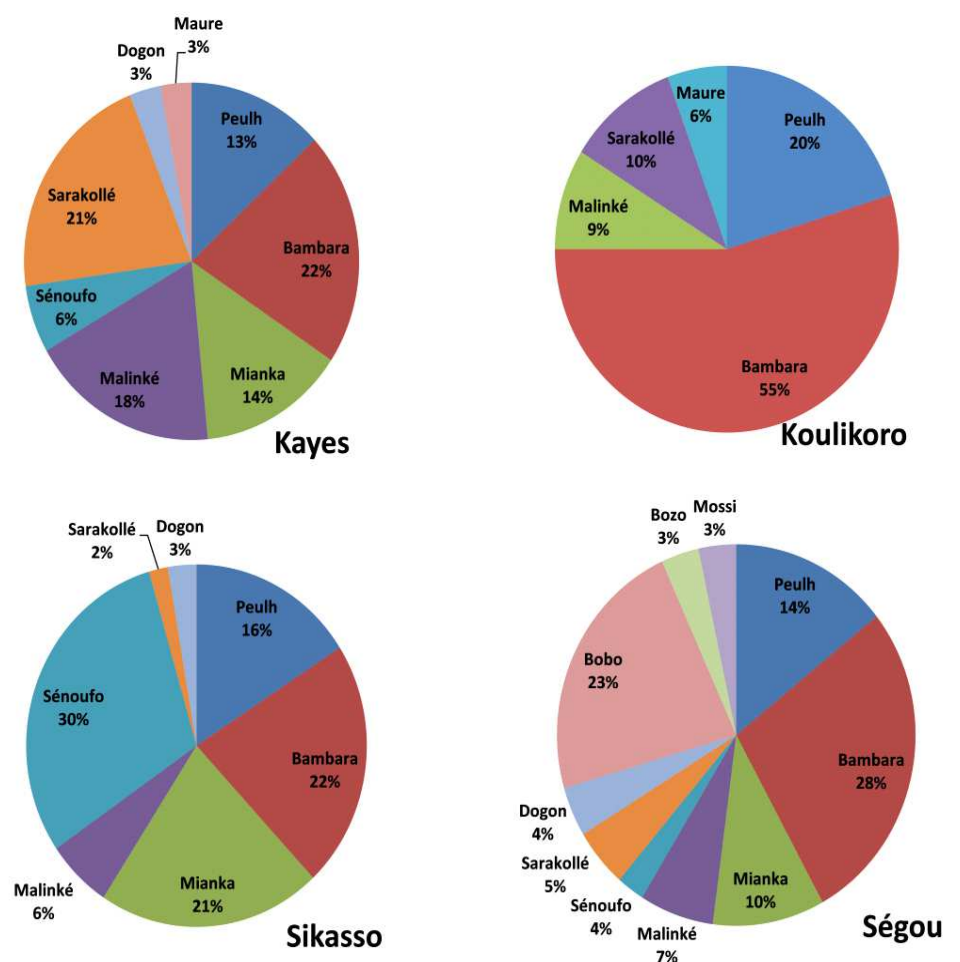

Figure 2: Répartition de la population enquêtée par groupe ethnique et par régions. 
Tableau 1: Répartition des répondants de l'enquête par sexe et par région.

\begin{tabular}{clcccc}
\hline \multicolumn{1}{c}{ Régions } & & Féminin & Masculin & Effectif total & Proportion* $(\%)$ \\
\hline Kayes & & & & & \\
& Bafoulabe & 4 & 27 & 31 & 6,8 \\
& Diéma & 10 & 30 & 40 & 8,8 \\
& Kita & 11 & 17 & 28 & 6,2 \\
& Total Kayes & 25 & 74 & 99 & 21,8 \\
\hline Koulikoro & & & & & \\
& Banamba & 11 & 20 & 31 & 6,8 \\
& Dioila & 4 & 36 & 40 & 8,8 \\
& Kolokani & 14 & 38 & 52 & 11,5 \\
& Total Koulikoro & 29 & 94 & 123 & 27,1 \\
\hline Sikasso & & & & & \\
& Bougouni & 11 & 24 & 35 & 7,7 \\
& Koutiala & 5 & 35 & 40 & 8,8 \\
& Yanfolila & 8 & 30 & 38 & 8,4 \\
& Total Sikasso & 24 & 89 & 113 & 24,9 \\
\hline Ségou & & & & & \\
& Bla & 8 & 29 & 37 & 8,1 \\
& San & 12 & 30 & 42 & 9,3 \\
& Konobougou & 18 & 22 & 40 & 8,8 \\
& Total Ségou & 38 & 81 & 119 & 26,2 \\
\hline Total global & & 116 & 338 & 454 & 100
\end{tabular}

\section{DISCUSSION}

Dans le but d'identifier les plantes sauvages consommées au sud du Mali, une enquête a été menée dans les régions de cette partie du pays. Au cours de cette enquête, 454 personnes ont été interrogées. Les données ont été collectées lors d'un passage transversal qui a permis d'identifier 87 plantes alimentaires. Une étude menée auprès des Malinkés de la région de Séguéla a recensé 75 plantes à fruits comestibles (Ambé 2001), cette étude avait été menée dans la zone pré-guinéenne avec une pluviométrie excédentaire favorisant la coexistence de plusieurs plantes à potentiel nutritif élevé. Dans le Natal, 33 espèces sont consommées en cas de famine, notamment les fruits de Carissa macrocarpa et de Bequaertiodendron natalense, et les racines de nombreuses espèces, en particulier Boscia albitrunca. Une étude anthropologique faite au Sénégal rappelle que les plantes sauvages jouent dans l'alimentation des Bassari (une ethnie du Sénégal) un rôle non négligeable, fournissant des fruits, des graines, des feuilles, des sèves, des bourgeons terminaux dont certains sont consommés tels, crus, en dehors des repas (Chastanet, 2010). 35 espèces de légumes feuilles consommées ont été répertoriées dans deux villages de la région de Pobè au Benin (Codjia et al., 2009).

Le baobab, le néré, le karité, le jujubier, et le dattier sauvage ont été les plantes les plus citées dans les sites enquêtés. Les résultats de cette présente étude ont montré que ces plantes sont connues par plus de 95\% des personnes enquêtées et consommées par plus de $90 \%$ d'entre elles. Ces résultats sont conformes à ceux d'Ambé (2001) qui a estimé les fréquences de reconnaissance et de consommation des mêmes plantes à plus de $90 \%$. Contrairement à ces résultats, Berge et al. (2005) ont identifié Zizyphus jujuba, Panicum laetum, Cenchrus biflorus et Boscia senegalensis comme plantes à fréquence d'utilisation élevées (plus de 60\%) dans le Gourma (situé au Nord du Mali). 
Tableau 2 : Liste globale des plantes recensées dans toutes les localités enquêtées.

\begin{tabular}{|c|c|c|c|c|c|}
\hline Noms scientifiques des plantes & Familles & $\begin{array}{l}\text { Nom Local } \\
\text { (Bamanan) }\end{array}$ & $\begin{array}{l}\text { Parties } \\
\text { utilisées }\end{array}$ & $\begin{array}{l}\text { Fréquence } \\
\text { de citation }\end{array}$ & $\begin{array}{c}\text { Numéro } \\
\text { d'herbier }\end{array}$ \\
\hline Adansonia digitata $\mathrm{L}$. & Malvaceae & Sira & $\mathrm{Fe}, \mathrm{Fr}, \mathrm{G}$ & $90 \%$ & 1513/DMT \\
\hline Aloe tenuior Haw. & Xanthorrhoeaceae & Ntole baga & $\mathrm{Fe}$ & $21 \%$ & $1005 / D M T$ \\
\hline Amaranthus graecizans L. & Amaranthaceae & Bouraboura ba & $\mathrm{Fe}$ & $16 \%$ & 0488/DMT \\
\hline Annona senegalensis Pers. & Annonaceae & Dangani & $\mathrm{Fe}$ & $30 \%$ & 0012/DMT \\
\hline Anthocleista djalonensis A.Chev. & Gentianaceae & Samaklo & $\mathrm{Fe}$ & $46 \%$ & 2318/DMT \\
\hline Balanites aegyptiaca $($ L.) Delile & Zygophyllaceae & Zèkènè & $\mathrm{Fe}, \mathrm{Fr}$ & $85 \%$ & 2015/DMT \\
\hline Bambusa vulgaris Schrad & Poaceae & Boh & $\mathrm{Fe}$ & $70 \%$ & 1904/DMT \\
\hline Bauhinia thonningii Schum. & Leguminosae & Niama & $\mathrm{Fe}$ & $8 \%$ & 2204/DMT \\
\hline Blighia sapida K. D. Koenig & Sapindaceae & Wara tiga & $\mathrm{Fe} \mathrm{Fr}$ & $65 \%$ & 1421/DMT \\
\hline Bombax costatum Pellegr. \& Vuillet. & Malvaceae & Boumboum & $\mathrm{Fe} \mathrm{Fl}$ & $47 \%$ & 1514/DMT \\
\hline Borassus aethiopum Mart. & Arecaceae & Sébé & $\mathrm{Fr}, \mathrm{G}$ & $86 \%$ & 2803/DMT \\
\hline Boscia senegalensis Lam & Capparaceae & Béré & $\mathrm{Fe}$ & $56 \%$ & 2784/DMT \\
\hline Calotropis procera (Aiton) Dryand. & Apocynaceae & Pompo pokolo & $\mathrm{Fe}$ & $28 \%$ & 2784DMT \\
\hline Senna italica Mill. & Leguminosae & Balabali & $\mathrm{Fe}$ & $10 \%$ & 0962/DMT \\
\hline Ceiba pentandra (L.) Gaertn. & Malvaceae & Banan & $\mathrm{Fe}, \mathrm{Fl}$ & $35 \%$ & $0134 / D M T$ \\
\hline Centaurea perrottetii DC. & Compositae & Gnokomégneki & $\mathrm{Fe}$ & $57 \%$ & 0658/DMT \\
\hline Ceratotheca sesamoides Endl. & Pedaliaceae & N'tekou & $\mathrm{Fe}$ & $12 \%$ & 808/DMT \\
\hline Cissus quadrangularis $\mathrm{L}$. & Vitaceae & Nofon & $\mathrm{Fe}$ & $42 \%$ & 1249/DMT \\
\hline Citrullus lanatus (Thunb) Matsum. \& Nakai & Cucurbitaceae & Zérénidjè & $\mathrm{Fe}$ & $35 \%$ & 2834/DMT \\
\hline Cochlospermum tinctorium Perrier ex A.Rich & Bixaceae & N'tilibara & $\mathrm{Fe}$ & $78 \%$ & 0375/DMT \\
\hline Cola cordifolia (Cav).R.Br. & Malvaceae & Tamba noko & $\mathrm{Fe}, \mathrm{Fr}$ & $83 \%$ & 2886/DMT \\
\hline Combretum lecardii Engl. \& Diels & Combretaceae & Demba foura & $\mathrm{Fe}$ & $68 \%$ & 1778/DMT \\
\hline Combretum micranthum G.Don. & Combretaceae & N'golobè & $\mathrm{Fe}$ & $90 \%$ & 2786DMT \\
\hline Commiphora africana A. (Rich) Engel. & Burseraceae & Baraganté & G & $87 \%$ & 0491/DMT \\
\hline Corchorus tridens $\mathrm{L}$. & Malvaceae & Zofon & $\mathrm{Fe}$ & $83 \%$ & 0832/DMT \\
\hline Cordia myxa $\mathrm{L}$. & Boraginaceae & N'tegué & $\mathrm{Fe}, \mathrm{Fr}$ & $14 \%$ & 1162/DMT \\
\hline Cordyla pinnata (A.Rich.) Milne-Redh. & Leguminosae & Dougoura & $\mathrm{Fe}, \mathrm{Fr}$ & $35 \%$ & 3754/DMT \\
\hline
\end{tabular}


N. DIARRA et al. / Int. J. Biol. Chem. Sci. 10(1): 184-197, 2016

\begin{tabular}{|c|c|c|c|c|c|}
\hline Crossopteryx febrifuga (Afzel. ex G.Don) Benth & Rubiaceae & Balembo & $\mathrm{Fe}, \mathrm{Fr}$ & $35 \%$ & 3049/DMT \\
\hline Detarium microcarpum G. et Perr. & Leguminosae & Tamba coumba & $\mathrm{Fe}, \mathrm{Fr}$ & $48 \%$ & 2765/DMT \\
\hline Digitaria horizontalis Willd. & Poaceae & Kononi ka fini & $\mathrm{Fe}$ & $8 \%$ & 2406/DMT \\
\hline Dioscorea alata $\mathrm{L}$. & Dioscoreaceae & Nianan & $\mathrm{R}$ & $4 \%$ & 1665/DMT \\
\hline Diospyros mespiliformis Hochst. ex A.DC. & Ebénaceae & Sounzounfin & $\mathrm{Fe}, \mathrm{Fr}$ & $24 \%$ & 0174/DMT \\
\hline Eclipta prostrata (L.) L. & Compositae & Mossofing & $\mathrm{Fe}$ & $55 \%$ & 2331/DMT \\
\hline Elaeis guineensis Jacq. & Arecaceae & N'teen & $\mathrm{Fe}, \mathrm{Fr}, \mathrm{G}$ & $47 \%$ & 2842/DMT \\
\hline Feretia apodanthera Delile & Rubiaceae & Djourasounkala & $\mathrm{Fe}$ & $44 \%$ & 1201/DMT \\
\hline Ficus capensis & Moraceae & Seré toro & $\mathrm{Fe}, \mathrm{Fr}$ & $65 \%$ & 0049/DMT \\
\hline Ficus dicranostyla Mildbr. & Moraceae & Namimatoro & $\mathrm{Fe}, \mathrm{Fr}$ & $13 \%$ & 0308/DMT \\
\hline Ficus glumosa Delile & Moraceae & N'gaba & $\mathrm{Fe}$ & $9 \%$ & 0565/DMT \\
\hline Ficus sycomorus L. (Miq) Steud. & Moraceae & Sou toro & $\mathrm{Fe}$ & $47 \%$ & 0139/DMT \\
\hline Ficus thonningii Blume. & Moraceae & Doubaley & $\mathrm{Fe}$ & $88 \%$ & 2933/DMT \\
\hline Gardenia aqualla Stapf \& Hutch & Rubiaceae & Bouré kè & $\mathrm{Fe}$ & $45 \%$ & 1306/DMT \\
\hline Gardenia erubescens Stapf \& Hutch & Rubiaceae & M'Bouré mousso & $\mathrm{Fe}, \mathrm{Fr}$ & $47 \%$ & 0775/DMT \\
\hline Grewia mollis Juss. & Malvaceae & Nogo nogo ba & $\mathrm{Fe}$ & $18 \%$ & 0235/DMT \\
\hline Cleome gynandra L. (Briq) & Cleomaceae & Nah sebé & $\mathrm{Fe}$ & $7 \%$ & 0750/DMT \\
\hline Hexalobus monopetalus (A.rich).E.et D. & Annonaceae & Vougagné & $\mathrm{Fe}$ & $41 \%$ & 0238/DMT \\
\hline Hibiscus sabdariffa $\mathrm{L}$. & Malvaceae & Dah & $\mathrm{Fe}, \mathrm{Fr}, \mathrm{G}$ & $42 \%$ & 0189/DMT \\
\hline Hyphaene thebaica Mart. & Arecaceae & Zimini & $\mathrm{Fr}$ & $13 \%$ & 03769/DMT \\
\hline Hyptis spicigera Lam. & Lamiaceae & Bénéfing & $\mathrm{Fr}$ & $28 \%$ & 0838/DMT \\
\hline Ipomoea cairica $(\mathrm{L}$.$) Sweet$ & Convolvulaceae & Foroko farakablé & $\mathrm{Fe}$ & $6 \%$ & 2807/DMT \\
\hline Ipomoea asarifolia (Desr.) Roem. \& Schult & Convolvulaceae & Forokofaraka & $\mathrm{Fe}$ & $19 \%$ & 0088/DMT \\
\hline Kigelia africana (Lam) Benth. & Bignoniaceae & Dedan ou yiriba & $\mathrm{Fe}$ & $12 \%$ & 2925/DMT \\
\hline Landolphia heudelotii A.DC. & Apocynaceae & Gueyi & $\mathrm{Fe} \mathrm{Fr}$ & $48 \%$ & 2454/DMT \\
\hline Lannea acida A.Rich. & Anacardiaceae & M'pekou guèlèni & $\mathrm{Fe}, \mathrm{Fr}$ & $33 \%$ & 1315/DMT \\
\hline Lannea velutina A Rich. & Anacardiaceae & Bakorompekou & $\mathrm{Fe}, \mathrm{Fr}$ & $21 \%$ & 1464/DMT \\
\hline Leptadenia lancifolia (Schumach. \& Thonn.) Decne (Pers.) Decne & Apocynaceae & Zongné & $\mathrm{Fe}$ & $5 \%$ & 1157/DMT \\
\hline Lippia chevalieri Moldenke & Verbenaceae & Nganiba kala & $\mathrm{Fe}$ & $85 \%$ & 2775/DMT \\
\hline Manilkara obovata (Sabine \& G.Don) J.H.Hemsl. & Sapotaceae & Manan & $\mathrm{Fe}$ & $2 \%$ & 0073/DMT \\
\hline Nymphaea lotus L. & Nymphaeaceae & N'gokou & $\mathrm{Fe}, \mathrm{Fr}$ & $18 \%$ & 3068/DMT \\
\hline
\end{tabular}


N. DIARRA et al. /Int. J. Biol. Chem. Sci. 10(1): 184-197, 2016

\begin{tabular}{|c|c|c|c|c|c|}
\hline Moringa oleifera Lam. & Moringaceae & N'gnoukou & $\mathrm{Fe}, \mathrm{Fr}$ & $14 \%$ & 1391/DMT \\
\hline Sarcocephalus latifolius (Sm.) E.A.Bruce. & Rubiaceae & Baro & $\mathrm{Fe}$ & $39 \%$ & 1117/DMT \\
\hline Nelsonia canescens (Lam) Spreng. & Acanthaceae & Kononi ka dolo & $\mathrm{Fe}, \mathrm{Fr}$ & $55 \%$ & 2282/DMT \\
\hline Neocarya macrophylla (Sabine) Prance ex F. White & Chrysobalanaceae & Wô & Fe, Fr, Tige & $51 \%$ & 1557/DMT \\
\hline Opilia amentacea Roxb. & Opiliaceae & korogouein & $\mathrm{Fe}$ & $52 \%$ & 1420/DMT \\
\hline Parinari curatellifolia Planch. ex Benth & Chrysobalanaceae & Toutou & $\mathrm{Fe}$ & $11 \%$ & 0085/DMT \\
\hline Parkia biglobosa (Jacq.) G.Don. & Leguminosae & Nérè & $F e, F r, G$ & $85 \%$ & 1802/DMT \\
\hline Pericopsis laxiflora (Baker) Meeuwen & Leguminosae & Koloklo & $\mathrm{Fe}$ & $60 \%$ & 2812/DMT \\
\hline Dicliptera paniculata (Forssk.) I.Darbysh... & Acanthaceae & Barakala & $\mathrm{Fe}$ & $11 \%$ & $1434 / D M T$ \\
\hline Portulaca oleraceae $\mathrm{L}$. & Portulacaceae & Missikoumbéré & $\mathrm{Fe}$ & $38 \%$ & 1934/DMT \\
\hline Raphionacme splendens subsp. bingeri (A.Chev.) Venter & Apocynaceae & M'pié & $\mathrm{R}$ & $4 \%$ & 1111/DMT \\
\hline Saba senegalensis (A. DC.) Pichon & Apocynaceae & Zaban & $\mathrm{Fe}, \mathrm{Fr}$ & $78 \%$ & 0082/DMT \\
\hline Senna obtusifolia (L.) H.S.Irwin \& Barneby & Leguminosae & Banikonoka tiga & $\mathrm{Fe}$ & $16 \%$ & 1533/DMT \\
\hline Scoparia dulcis L. & Plantaginaceae & Timitimini & $\mathrm{Fe}$ & $79 \%$ & 1966/DMT \\
\hline Sclerocarya birrea (A. Rich). Hochst. & Anacardiaceae & N'gounan & $\mathrm{Fe}$ & $15 \%$ & 2355/DMT \\
\hline Flueggea virosa (Roxb. ex Willd.) Royle & Phyllanthaceae & N'tièlè & $\mathrm{Fe}$ & $12 \%$ & 2819/DMT \\
\hline Solanum incanum $\mathrm{L}$. & Solanaceae & Bah N'gôyo & $\mathrm{Fe}$ & $3 \%$ & 2689/DMT \\
\hline Spondias mombin $\quad \mathrm{L}$. & Anacardiaceae & Minkon & $\mathrm{Fe}, \mathrm{Fr}$ & $17 \%$ & 1148/DMT \\
\hline Stachytarpheta indica (L.) Vahl & Verbenaceae & Bassakou & $\mathrm{Fe}$ & $2 \%$ & 0883/DMT \\
\hline Strychnos innocua Delile. & Loganiaceae & Gankoro & $\mathrm{Fe}$ & $10 \%$ & 0558/DMT \\
\hline Tamarindus indica $\quad \mathrm{L}$. & Leguminosae & Tomi & $\mathrm{Fe}, \mathrm{Fr}$ & $68 \%$ & 2902/DMT \\
\hline Uraria picta (Jacqw.) DC. & Leguminosae & Alagno & $\mathrm{Fe}$ & $22 \%$ & 0824/DMT \\
\hline Vitex doniana Sweet. & Lamiaceae & Koroba & $\mathrm{Fe}$ & $21 \%$ & 0065/DMT \\
\hline Vitex mandiensis Oliv. & Lamiaceae & Koronifing & $\mathrm{Fe}, \mathrm{Fr}$ & $25 \%$ & 0301/DMT \\
\hline Vitellaria paradoxa C.F.Gaertn & Sapotaceae & Chi & $\mathrm{Fe}, \mathrm{Fr}, \mathrm{G}$ & $88 \%$ & 0137/DMT \\
\hline Waltheria indica $\mathrm{L}$. & Malvaceae & Dabada & $\mathrm{Fe}$ & $55 \%$ & 2811/DMT \\
\hline Ximenia americana $\mathrm{L}$. & Olacaceae & Ntonkè & $\mathrm{Fe}$ & $65 \%$ & 0764/DMT \\
\hline Ziziphus jujuba Mill. & Rhamnaceae & Tomonon & $\mathrm{Fe}, \mathrm{Fr}$ & $39 \%$ & 2369/DMT \\
\hline Ziziphus mucronata Willd. & Rhamnaceae & souroukou tomonon & $\mathrm{Fe}$ & $16 \%$ & 2222/DMT \\
\hline
\end{tabular}


Ceci démontre la variabilité des espèces en fonction des zones climatiques et les différences au niveau des habitudes alimentaires entre les populations du Nord et celles du Sud. En effet, l'importance d'une plante alimentaire sauvage varie en fonction de l'habitude alimentaire de la population en présence.

Les plantes les moins consommées sont pour la plupart des herbacées ou des plantes annuelles, donc disponibles pendant une courte période de l'année. Les parties les plus consommées de ces plantes sont les fruits à l'état naturel ou cuits suivi des feuilles qui sont consommées après cuisson soit comme sauce et/ou compléments alimentaires. La valeur nutritive de l'ensemble de ces plantes n'est pas bien connue. Cependant, certaines d'entre elles peuvent être utilisées comme compléments alimentaires. Leur teneur en éléments minéraux fait que la plupart de ces plantes est nécessaire pour l'équilibre alimentaire surtout pendant les périodes de soudure.

Les fruits du baobab, du néré, du Saba senegalensis et du karité sont transformés pour être disponibles toute l'année (sous formes de jus, de sirop, de poudre, ou de fruits secs).

Les fruits du Néré sont fort recherchés par les populations rurales. Ils mangent la pulpe jaune farineuse qui souvent leur tient de toute autre nourriture, surtout quand ils voyagent. Elle est fort bonne, nourrissante et d'un goût de pain d'épices sucrée et très agréable, mélangé au miel, elle serait utilisée comme un moyen de lutte contre le paludisme. D'autre part, les graines de Parkia biglobosa sont essentiellement utilisées dans la préparation d'une moutarde très recherchée appelée «soumbala ». Ce produit est très riche en protéines avec une teneur pouvant parfois atteindre $40 \%$ (Ouoba et al., 2005). Cette moutarde qui est issue d'une longue cuisson et de la fermentation des graines fait l'objet de transactions dans les différents marchés de la sous-région car elle constitue le principal ingrédient des sauces. Au Burkina Faso, plus de $90 \%$ des ménages utilisent le soumbala (Ouoba et al., 2005).

Les plantes comme Digitaria horizontalis (graines), Dioscorea alata (tubercules ou racines), Gynandropsis gynandra (feuilles), Leptadenia lancifolia (fruits) et Raphionachme daroni (tubercules) sont surtout utilisées pendant les périodes de famine et de soudure (Sène, 2000). Dans la vallée de la Luangwa (Zambie), les végétaux sauvages jouent un rôle important dans l'alimentation, en particulier pendant la période de soudure. Dans le village de Mukupu (Zambie), par exemple, les légumes sauvages entrent dans la composition de $42 \%$ des repas en septembre, contre $10 \%$ pour les légumes cultivés pendant cette même période; en revanche, en juin les légumes sauvages sont utilisés dans $7 \%$ seulement des plats (contre plus de $50 \%$ pour les variétés cultivées) tandis qu'au Botswana, les Bochimans San tirent de l'eau potable de nombreuses plantes, en particulier les tubercules de Raphionacme burkei et de Coccinea rehmannii (van Wyk et Gericke, 2007; Lapante, 2009)

L'amande du karité est utilisée pour la fabrication du beurre de karité, une source oléagineuse importante pour les préparations culinaires. Le beurre est utilisé plus fréquemment dans la préparation des sauces (89\%) que les autres huiles végétales (Elias et Carney, 2004). Par ailleurs, le beurre de karité entre dans la fabrication de chocolat, de savons modernes et de produits cosmétiques.

Les feuilles de baobab fraîches ou séchées sont riches en provitamine A, protéines, glucides, calcium, phosphore et acides aminés (Neba, 2009; Leroy et al., 2013; Rahul et al., 2015). Elles sont utilisées principalement comme légumes de sauces qui accompagnent la pâte de céréale appelé tô (Guimbo et al., 2012). Elles sont aussi utilisées pour la préparation du couscous ou 
$\mathrm{du}$ fonio afin de faciliter leur ingestion en les rendant un peu gluants. Comme les feuilles, la pulpe blanchâtre des fruits a également une utilisation alimentaire; elle a un goût acidulé et sucré, et après dissolution dans l'eau, elle donne une boisson aigre, les femmes l'utilisent également pour la préparation de bouillie (Neba, 2009; Leroy et al., 2013; Rahul et al., 2015).

Les fruits en maturité de Neocarya macrophylla sont ramassés pour être stockés et consommés durant toute l'année. Leur consommation sert de prévention contre le paludisme, d'où sa recommandation par les personnes âgées durant la période allant de juillet à novembre (pic du paludisme) (Balla et al., 2008).

Hormis les plantes populairement connues comme le baobab, le néré, le karité, le jujubier, et les balanites, d'autres comme Dioscorea alata et Moringa oleifera sont d'un apport très important. Dioscorea alata mal préparé peut-être toxique, ce qui fait que sa récolte et la préparation est confiée aux vieilles femmes. Cependant, bien préparé, il est largement utilisé dans l'alimentation dans certaines zones d'enquêtes surtout pendant la période ou les récoltes viennent à manquer. Les tubercules de cette plante sont bien lavés et mis en marmite avec de l'eau pour une cuisson pendant de longues heures en renouvelant de temps en temps l'eau de cuisson, ce qui permettrait de les débarrasser de leur toxines. Ils sont ensuite écrasés avec un peu de beurre de karité et un peu de sel. La consommation d'une petite quantité de ce plat ainsi préparé permet de résister longtemps à la faim tout en restant actif. Le mode de cuisson a un impact sur la toxicité et la valeur nutritionnelle de certaines plantes. Vodouhe et al. (2012) ont démontré que la cuisson à l'eau bouillante augmentait les teneurs en protéines et en lipides de Amaranthus hybridus, celles en lipides de Solanum macrocarpum et celles en protéines de Ocimum gratissimum, trois légumes acclimatés au Benin. $\mathrm{Si}$ une éventuelle toxicité était liée à l'un de ces composés, alors le mode de cuisson aurait forcément un impact sur le degré de cette toxicité.

Les résultats de ce travail ont aussi montré que Cleome gynandra L. (Briq) est aussi d'un grand apport nutritionnel. Ses feuilles sont consommées dans les sauces comme condiments ou seules comme aliment après avoir été bouillies dans de l'eau puis assaisonnées avec un peu de sel et de beurre de karité. Aussi, les fruits murs bien sucrés de Hexalobus monopetalus sont beaucoup appréciés par de nombreux bergers, ce qui leur permet de mieux résister à la faim. Des milliers d'espèces de fruits sauvages sont consommés dans le monde entier. Les fruits sont particulièrement riches en minéraux et vitamines et confèrent parfois à l'organisme des quantités élevées de calories. Les fruits sont normalement consommés frais, comme collation ou aliment d'appoint. Les fruits récoltés dans les forêts sont également largement utilisés pour la fabrication de boissons, la bière en particulier. En Inde, il a été estimé que 50 millions de ménages enrichissent leur régime alimentaire avec les fruits cueillis dans la forêt et la brousse environnante (FAO, 2011).

Les fleurs séchées de Bombax costatum sont disponibles en fin de saison hivernale ou en début de la saison sèche. Ces fleurs séchées sont utilisées dans la préparation d'une sauce qui a la réputation de lutter contre la constipation. La consommation des produits de cueillette est rentrée dans les habitudes alimentaires un peu partout au Mali. La population du Gourma malien utilise également grand nombre de plantes sauvages dans la préparation des repas (Berge et al., 2005).

Les résultats de ce travail démontrent clairement l'importance des plantes sauvages dans la vie des populations rurales. Ces plantes et leurs produits de transformation sont non seulement utilisés comme sources 
d'aliment lorsque les réserves de grains sont épuisées, mais aussi représentent une source de génération de revue pour les communautés. Cependant, peu d'études sont menées sur l'impact de l'utilisation de ces plantes sur l'alimentation et l'économie des populations rurales. Des études scientifiques démontrent la valeur nutritionnelle de certaines plantes comme le néré et le karité, mais la majorité des plantes citées lors de ces enquêtes sont de bons candidats pour une étude approfondie de leur valeur nutritive. Les résultats de ces études permettront la fabrication d'aliments locaux pour la prise en charge de la malnutrition, ce qui contribuera à la valorisation des espèces concernées et leur protection contre la disparition.

En perspective, une étude similaire doit être menée dans les régions nord du Mali afin de répertorier les plantes les plus répandues à l'échelle nationale et les plantes spécifiques à chaque région. Cette approche permettra d'avoir un répertoire complet de l'ensemble des plantes sauvages alimentaires $\mathrm{du}$ Mali et la réalisation d'une base de données et un herbier national au sein du Département de Médecine Traditionnelle.

Enfin, la détermination de la composition chimiques et du dosage des macromolécules, vitamines et éléments minéraux permettra de sélectionner les plantes à haut potentiel nutritif pour leur protection et pour leur vulgarisation.

\section{Conclusion}

Cette étude a permis de montrer l'existence d'une grande diversité de plantes sauvages alimentaires dans les régions sud du Mali. En plus, elle a montré que la consommation d'un fort pourcentage de ces plantes est commune à toutes les régions. Les résultats des analyses ont montré l'importance capitale des plantes sauvages pour les populations rurales pendant la période de soudure où les réserves en céréales sont épuisées. Ces plantes méritent d'être valorisées afin de lutter contre leur disparition à travers une domestication par la maîtrise des techniques culturales.

\section{CONFLITS D'INTÉRÊTS}

Les auteurs déclarent l'absence de conflit d'intérêt.

\section{CONTRIBUTIONS DES AUTEURS}

ND a élaboré le protocole, mené l'enquête sur le terrain récolté les échantillons de plantes, analysé les données et rédigé le manuscrit. AT a participé à l'élaboration du protocole et du questionnaire, à la rédaction et la correction du manuscrit. $\mathrm{AD}$ a participé à l'enquête sur le terrain et à la récolte des échantillons. MW a participé à l'analyse des données et à la rédaction de la partie discussion. CD a corrigé les noms scientifiques de plantes. DD a corrigé le protocole et le manuscrit final et a participé à l'analyse des données.

\section{REMERCIEMENTS}

Nous remercions l'équipe du Département de Médecine Traditionnelle pour son support dans l'identification des plantes, les associations des tradipraticiens de santé des différentes localités ainsi que toutes les autres personnes ressources.

\section{RÉFÉRENCES}

Ambé GA. 2001 Les fruits sauvages comestibles des savanes guinéennes de Côte-d'Ivoire: état de la connaissance par une population locale, les Malinké. Biotechnol. Agron. Soc. Environ., 5(1): 43-58. http://www.pressesagro.be/base/ index.php/base/article/view/148

Balla A, Baragé M, Larwanou M et Adam T. 2008. Le savoir-faire endogène dans la valorisation alimentaire des fruits du pommier du Cayor (Neocarya macrophylla Sabine) au Niger. Bull. Rech. Agron. Bénin., 59: 1-8. http://www.slire.net/document/705 
Berge G, Diallo D, Hveem B. 2005. Les Plantes Sauvages du Sahel Malien, les Stratégies d'Adaptation à la Sécheresse. Editions Karthala: Paris.

Chastanet M. 2010 Couscous 'à la sahélienne' (Sénégal, Mali, Mauritanie) », In Couscous, Boulgour et Polenta. Transformer et Consommer les Céréales dans le Monde, Franconie $\mathrm{H}$, Chastanet M, Sigaut F (eds). Karthala édition: Paris; 149-187.

Codjia JTC, Vihotogbe R, Lougbegnon T. 2009. Phytodiversité des légumes-feuilles locales consommées par les peuples Holli et Nagot de la région de Pobè au sud-est du Bénin. Int. J. Biol. Chem. Sci., 3(6): 1265-1273. DOI: http://dx.doi.org/ 10.4314/ijbcs.v3i6.53145

DRPS. 2000. (Direction Régionale du Plan et de la Statistique du Mali) Enquête Agricole De Conjoncture. Drsp Édition : Bamako.

Easton P, Ronald M. 2000. Les Femmes et la Biodiversité Végétale en Afrique. Banque mondiale Note: Washington.

Elias M, Carney J. 2004. La filière féminine du karité: productrices burkinabè, "écoconsommatrices" occidentales et commerce équitable. Cah. Géogr. Québ., 48(133): 71-88. DOI : http://dx.doi.org/ 10.7202/009763ar

FAO 2011. Les forêts au service de la nutrition et de la sécurité alimentaire. http://www.fao.org/docrep/014/i2011f/i20 $11 \mathrm{f00.pdf}$

INSTAT 2009. Institut National de la Statistique Mali. Commissariat à la Sécurité Alimentaire, Projet de Mobilisation des Initiatives en Matière de Sécurité Alimentaire au Mali. INSTAT édition: Bamako.

Guimbo ID, Barage M, Douma S. 2012. Etudes préliminaires sur l'utilisation alimentaire des plantes spontanées dans les zones périphériques du parc W du Niger. Int. J. Biol. Chem.
Sci., 6(6): 4007-4017. DOI: http://dx.doi.org/10.4314/ijbcs.v6i6.12

Laplante J. 2009. Plantes médicinales, savoirs et société: vue des rastafaris sud-africains. Drog. Sant. Sociét., 8(1): 93-121. DOI : http://dx.doi.org/10.7202/038917ar

Leroy M. Derroire J. Vendé T. 2013. La gestion durable des forêts tropicales de l'analyse critique du concept à l'évaluation environnementale des dispositifs de gestion. La gestion durable des forêts tropicales. http://www.afd.fr/jahia/webdav/site/afd/s hared

Lulekal E, Asfaw Z, Kelbessa E, Van Damme P. 2011. Wild edible plants in Ethiopia: a review on their potential to combat food insecurity. Afrik. Foc., 24(2): 71-121. http://hdl.handle.net/1854/LU-2017166

Neba NE. 2009. Management of woody plants in indigenous land use systems of the Sahel: Example of north Cameroon. Int. NGO J. 4(11): 480-490. http://www.academicjournals.org/journal/ INGOJ/article-stat/4D8FC1C40372

Ouoba LII, Diawara B, Annan NT, Poll L, Jakobsen M. 2005. Volatile compounds of Soumbala, a fermented African locust bean (Vitellaria paradoxa) food condiment. J App. Microb., 99: 14131421. DOI: http://dx.doi.org/10.1111/ j.1365-2672.2005.02722.x

Rahul J, Jain MK, Singh SP, Kamal RK, Anuradha A, Naza A, Gupta AK, Mrityunjaya SK. 2015. Adansonia digitata L. (baobab): a review of traditional information and taxonomic description. Asian Pacif. J. Trop. Biomed., 5(1): 79-84. DOI : http://dx.doi.org/10.1016/s22211691(15)30174.x

Roudart L. 2007. Alimentation et malnutrition dans le monde. Eco. Hum., 380: 9-25.

Sène EH. 2000. Forêts et sécurité alimentaire en Afrique. La place de la foresterie dans le Programme spécial pour la sécurité 
alimentaire de la FAO. In : Forêts, sécurité alimentaire et moyens de subsistance durables, archives documents FAO.

http://www.fao.org/docrep/x7273f/x7273f 00.htm

SNPA/BD.2000. Stratégie Nationale en matière de biodiversité biologique au Mali 1: 123. http://www.onmali.org/pdf/snpav1.pdf

Tollens E. 2003. L'état actuel de la sécurité alimentaire en R.D. Congo: Diagnostic et perspectives Working Paper, $\mathrm{n}^{\circ} 77$ Département d'Economie Agricole et de l'Environnement, Katholieke Universiteit Leuven, http://www.agr.kuleuven.ac.be/ aee/clo/wp/tollens2003a.pdf van Wyk BE, Gericke N. 2007. People's Plants, A Guide to Useful Plants of Southern Africa. Priza: Pretoria.

Vodouhe S, Dovoedo A, Anihouvi VB, Tossou RC. 2012. Influence du mode de cuisson sur la valeur nutritionnelle de Solanum macrocarpum, Amaranthus hybridus et Ocimum gratissimum, trois légumes feuilles traditionnels acclimatés au Bénin. Int. J. Biol. Chem. Sci., 6(5) 1926-1937. DOI: http://dx.doi.org/ 10.4314/ijbcs.v6i5.3

Wentholt W, Dembélé ARK, Diallo M. 2001. Genre et Recherche Agricole au Mali. IER KIT Publishers: The Netherlands. 